\title{
Interaktion in ökologieorientierten Unternehmensnetzwerken
}

\author{
In ökologieorientierten Unternehmensnetzwerken organisieren sich Akteure aus \\ Wirtschaft und Gesellschaft, um kooperativ ökologische Verbesserungen anzu- \\ streben. Ungeklärt ist aber bislang, wie sich der Dualismus von Markt und Ge- \\ sellschaft auf die Effizienz der Netzwerkarbeit auswirkt. Hemmen oder be- \\ fruchten sich markt- und gesellschaftsbezogene Interessen gegenseitig?
}

$\mathrm{Z}$ Von Adina Herde ur Untersuchung der Dynamiken innerhalb von ökologieorientierten Unternehmensnetzwerken eignet sich der International Marketing and Purchasing-Ansatz (IMP). Bei weitem nicht so bekannt wie die Transaktionskostentheorie, rückt der IMP-Ansatz mit Interaktion, Macht und Abhängigkeit die entscheidenden Variablen für die Analyse von Öko-Netzwerken in den Mittelpunkt. Praktische Anknüpfungspunkte liefert ein aktuelles Projekt des Instituts für ökologische Wirtschaftsforschung (IÖW): Die Entwicklung einer nachhaltigen Kundenkarte im Rahmen eines lokalen Akteursnetzwerkes (1).

\section{- Idealtypen: markt- und gesell- schaftsbezogene Netzwerke}

Die Forschung im Bereich der ökologieorientierten Unternehmensnetzwerke ist noch nicht sehr weit fortgeschritten, schließlich handelt es sich hier um eine recht neue Erscheinung (2). Ökologieorientierte Unternehmensnetzwerke können zwei Idealtypen zugeordnet werden: marktbezogenen oder gesellschaftsbezogenen Netzwerken.

Marktbezogene Unternehmensnetzwerke werden gebildet, um die Wettbewerbsfähigkeit von ökonomischen Akteuren zu erhöhen und die Marktbedingungen zu beeinflussen. Diese zwei Punkte sind auch in Netzwerken mit einer ökologischen Ausrichtung nicht zu unterschätzen. Unternehmen in ökologieorientierten Netzwerken verfügen aufgrund ihrer geringen Erfahrung hinsichtlich der Produktion, des Vertriebes und der Bereitstellung von umweltverträglichen Produkten und Dienstleistungen nur über begrenztes Wissen. Deshalb ist die Interaktion mit anderen Akteuren umso wichtiger, um die Unsicherheit des Marktes zu kompensieren und die noch unbeständigen
Marktbedingungen zu beeinflussen. Typische marktbezogene Netzwerke sind regionale Entsorgungsringe, in denen bis zu 300 Unternehmen Produktabfälle gemeinsam entsorgen, um ihre Entsorgungskosten zu reduzieren.

Die Aufgabe von gesellschaftsbezogenen Unternehmensnetzwerken liegt darin, auf Anspruchsgruppen zu reagieren, Konflikte beizulegen und die gesellschaftspolitische Diskussion in Sinne einer zunehmenden Ökologisierung zu beeinflussen. Unternehmen beteiligen sich an solchen Netzwerken, um ihr Image in der Öffentlichkeit aufzubessern, neue Marktnischen zu besetzen und in Marktnischen für einen Nachfrageschub zu sorgen. PR-Aktionen sind ein wichtiges Strategieelement, um gesellschaftsbezogenen Netzwerke bekannt zu machen. So verteilen zum Beispiel die in einem ökologieorientierten Netzwerk zusammengeschlossenen Großunternehmen Coca-Cola, McDonald's, Esso und Aral Müllbeutel an die Bevölkerung, um auf ihre Aktion ,Saubere Landschaft" aufmerksam zu machen.

Der IMP-Ansatz setzt sich aus den Variablen $A k$ teure, Interaktionsprozess, Umwelt und Atmosphäre zusammen. Spezielle Netzwerkbeziehungen, das heißt Lernen, Investitionen, Anpassungen, Vertrauen/Engagement und Distanz, ergänzen das Modell (vgl. Abb. 1).

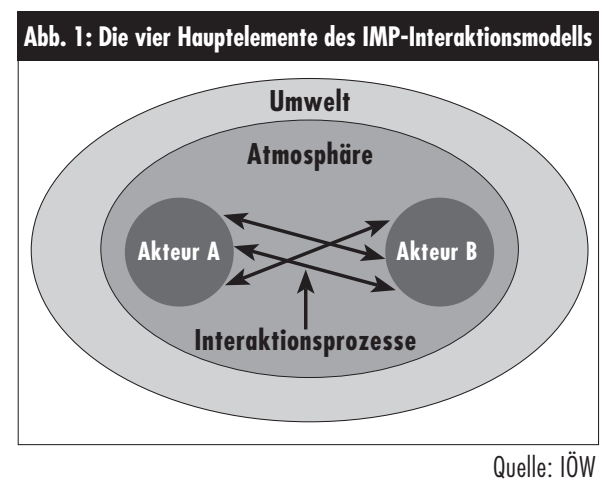

Im Rahmen des IÖW-Projektes zur Einführung einer nachhaltigen Kundenkarte wurde der IMPAnsatz praktisch angewendet. Die Ergebnisse der Projektuntersuchung, durchgeführt mittels leitfadengestïtzter Interviews, flossen in die Typologie von ökologieorientierten Unternehmensnetzwerken ein (siehe Tabelle 1).

\section{- Öko-Netzwerke im Spannungsfeld}

Die Analyse hat Folgendes zu Tage gebracht: Da sich in den meisten ökologieorientierten Unternehmensnetzwerken sowohl marktliche als auch gesellschaftliche Akteure befinden, kann es durchaus zu Interessenkonflikten kommen. Dass in marktbezogenen Netzwerken gewöhnlich mehr marktliche als gesellschaftliche Akteure organisiert sind, muss nicht automatisch heißen, dass nur marktbezogene Akteure zu den Schlüsselakteuren des Netzwerkes gehören. Gesellschaftlichen Akteuren, insbesondere aus dem politischen Umfeld, wird häufig eine große Autorität innerhalb des Netzwerks zugeschrieben, was sich auch auf ihre Netzwerkposition positiv auswirkt. Genauso kann in gesellschaftsbezogenen Netzwerken nicht zwangsläufig vorausgesetzt werden, dass nur gesellschaftliche Akteure als Schlüsselakteure fungieren. Dies führt zu einem Dualismus von Markt und Gesellschaft, der in den einzelnen ökologieorientierten Unternehmensnetzwerken unterschiedlich ausgeprägt sein kann.

Es ist durchaus anzunehmen, dass sich marktund gesellschaftsbezogene Interessen gegenseitig befruchten können und daher zu einer effizienten Netzwerkarbeit beitragen. Ein Mechanismus, um Interessenkonflikte in ökologieorientierten Unternehmensnetzwerken zu entschärfen und die Akteure zu einer ergiebigen Netzwerkarbeit zusammenzuführen, scheint die Herausbildung starker Netzwerkpositionen zu sein. Die Schlüsselakteure, die über diese starken Netzwerkpositionen verfïgen, übernehmen in der Regel koordinierende Funktionen, was angesichts der Unterschiedlichkeit der Akteure auch wichtig erscheint, um die verschiedenen Interessen unter einen Hut zu bringen. Die Schlüsselakteure im WeitergebenNetzwerk, darunter das IÖW, übernehmen neben der Moderation und Koordination durch interne Dokumentation und wissenschaftliche Begleitung das ,Sichtbarmachen“ der Netzwerkentwicklung, um die Zusammenarbeit besser strukturieren zu können. 
Tabelle 1: Interaktion in ökologieorientierten Unternehmensnetzwerken

Marktbezogene Netzwerke

Gesellschaftsbezogene Netzwerke

Interaktionsbeziehungen

\begin{tabular}{|l|l|l|}
\hline Akteure & $\begin{array}{l}\text { Vornehmlich marktliche Akteure } \\
\text { (z.B. Unternehmen), aber auch } \\
\text { gesellschaftliche Akteure (z.B. } \\
\text { Umweltorganisationen, Behörden) }\end{array}$ & $\begin{array}{l}\text { Marktliche und gesellschaftliche } \\
\text { Akteure }\end{array}$ \\
\hline Interaktionsprozesse & $\begin{array}{l}\text { Austausch von Informationen, Pro- } \\
\text { dukten, Dienstleistungen, Finanzen }\end{array}$ & $\begin{array}{l}\text { Informationsaustausch als dominante } \\
\text { Austauschform }\end{array}$ \\
\hline Wichtigste Umwelffaktoren & $\begin{array}{l}\text { Markt, Gesetze und staatliche } \\
\text { Förderung }\end{array}$ & \begin{tabular}{l} 
Gesellschaftliche Anspruchsgruppen \\
\hline Atmosphäre
\end{tabular} \\
\hline & $\begin{array}{l}\text { Keine ausgeprägten Macht- und Abhängigkeitsbeziehungen, aber Tendenz zur } \\
\text { Bildung von Schlüsselakteuren, die Moderations- und Koordinationsaufgaben } \\
\text { übernehmen }\end{array}$ \\
\hline
\end{tabular}

Spezielle Netzwerkbeziehungen

\begin{tabular}{|l|l|l|}
\hline Lernen & $\begin{array}{l}\text { Erfahrungsaustausch statt Wissensver- } \\
\text { mittlung, Ausbau der Kernkompe- } \\
\text { tenzen und Innovationen höchstens in } \\
\text { Produktionsnetzwerken }\end{array}$ & $\begin{array}{l}\text { Wissensvermittlung nach außen statt } \\
\text { Lernen voneinander }\end{array}$ \\
\hline Investitionen & $\begin{array}{l}\text { Kalkulierung der Kosten schwierig; ökologischen Zielen stehen ökonomische } \\
\text { Ziele gegenüber, wobei sich marktbezogene Netzwerke noch eher rentieren } \\
\text { müssen als gesellschaftsbezogene Netzwerke }\end{array}$ \\
\hline Anpassungen & $\begin{array}{l}\text { Anpassung der Produktionsabläufe } \\
\text { und Kommunikationsstrukturen }\end{array}$ & $\begin{array}{l}\text { Anpassung der Kommunikationsstruk- } \\
\text { turen }\end{array}$ \\
\hline Vertrauen und Engagement & Ausmaß an Vertrauen und Engagement in jedem Netzwerk individuell verschieden \\
\hline Distanz & $\begin{array}{l}\text { Technologische Distanz nur in Produk- } \\
\text { tionsnetzwerken relevant }\end{array}$ & $\begin{array}{l}\text { Soziale Distanz durch Unterschied- } \\
\text { lichkeit der Akteure }\end{array}$ \\
\hline
\end{tabular}

Quelle: IÖW

\section{Kritik am IMP-Ansatz}

Zunächst scheint es , als könnte der IMP-Ansatz der komplexen Realität von Unternehmensnetzwerken gerecht werden, da er andere Netzwerkansätze wie die Transaktionskostentheorie und die Theorie des sozialen Austausches in seine Betrachtung einbezieht und revidiert. Seine Stärke liegt darin, dass er soziale und ökonomische Aspekte miteinander zu verbinden versucht und nicht - wie in den anderen Theorien - getrennt betrachtet. Außerdem beschreibt er die Rolle von Schlüsselakteuren, die in ökologieorientierten Netzwerken als Vermittler zwischen marktlichen und gesellschaftlichen Interessen auftreten, sehr detailliert.

Beschäftigt man sich jedoch genauer mit dem IMPAnsatz, dann fällt auf, dass er auf einer Reihe von Prämissen basiert, die keinem gemeinsamen Erklärungszusammenhang entspringen. Daher gleicht er
Von vielen Seiten wird mittlerweile die inflationäre Verwendung des Netzwerkbegriffs beklagt. Keiner der zahlreichen Netzwerkansätze kann bisher der komplexen Organisationsform Netzwerk gerecht werden. Auch der IMP-Ansatz kann dies nur teilweise. Das IÖW-Projekt zeigt, dass die Interaktionsprobleme zwischen gesellschaftlichen und marktlichen Akteuren mit dem IMP-Ansatz sehr gut erfasst werden können. Ursprünglich war das Projekt als Tauschring konzipiert worden. Diese eher gesellschaftliche Zielsetzung wurde zugunsten der Kundenkarte aufgegeben, um mehr Wirtschaftlichkeit zu erreichen. Daraufhin kam es zu Unstimmigkeiten innerhalb des Netzwerkes: die gesellschaftlichen Akteure sahen ihre Interessen in dem nun marktbezogenen Netzwerk nicht mehr vertreten und zogen sich teilweise zurück. Die Umkonzeptionierung hat allerdings Erfolg: bisher wurden knapp 1000 Kundenkarten verkauft. Schwieriger gestaltet sich hingegen die Beschreibung der Macht- und Abhängigkeitsbeziehungen bei „Weitergeben“ (3). Dies liegt allerdings nicht ausschließlich an dem abstrakten Begriff der ,Atmosphäre“, sondern auch am knapp bemessenen zeitlichen Horizont der Untersuchung. Für andere Studien erscheint es ebenfalls sinnvoll, dass statt der bisherigen Querschnittstudien langfristig angelegte Intensivfallstudien durchgeführt werden.

\section{Anmerkungen}

(1) Einen Überblick über das Projekt gibt Scholl, G. : Von Tauschringen und Kundenkarten: Nachhaltige Dienstleistungsinnovation im lokalen Netzwerk. In: Ökologisches Wirtschaften 1/2003, S. 30f. Weitere Informationen unter www.weitergeben.info

(2) Zu den aktuellsten Veröffentlichungen gehören zwei Dissertationen: Störmer, E.: Ökologieorientierte Unternehmensnetzwerke, München 2001; Tischer, M.: Unternehmenskooperation und nachhaltige Entwicklung in der Region, Marburg 2001.

(3) Ausführlicher in Herde, A.: Interaktion in ökologieorientierten Unternehmensnetzwerken: Der interaktionistische Netzwerkansatz der IMP Group, Berlin 2003 (unveröffentlichte Diplomarbeit).

\section{Die Autorin}

Adina Herde war Diplomandin im Forschungsfeld Ökologische Produktpolitik am Institut für ökologische Wirtschaftsforschung.

Kontakt: Schönhauser Allee 125, 10437 Berlin, E-Mail: adina.herde@rz.hu-berlin.de. 
(c) 20I0 Authors; licensee IÖW and oekom verlag. This is an article distributed under the terms of the Creative Commons Attribution Non-Commercial No Derivates License (http://creativecommons.org/licenses/by-nc-nd/3.o/), which permits unrestricted use, distribution, and reproduction in any medium, provided the original work is properly cited. 